\title{
Density of states determination from steady-state photocarrier grating measurements
}

\author{
J. A. Schmidta) \\ INTEC (UNL-CONICET), Güemes 3450, 3000 Santa Fe, Argentina \\ FIQ (UNL), Santiago del Estero 2829, 3000 Santa Fe, Argentina \\ C. Longeaud \\ Laboratoire de Génie Electrique de Paris, (UMR 8507 CNRS) Ecole Supérieure d'Electricité, \\ Université Paris VI et XI, Plateau de Moulon, 91192 Gif-sur-Yvette Cedex, France
}

(Received 7 June 2004; accepted 14 September 2004)

\begin{abstract}
We present a method to obtain the density of states (DOS) of photoconductive insulators based on steady-state photocarrier grating (SSPG) measurements. A simple expression-relating the DOS at the electron quasi-Fermi level to measurable quantities-is deduced by performing suitable approximations from the analytical solution of the generalized equations that describe the SSPG experiment. The validity of the approximations and the applicability of the final expression are verified from numerical simulations of the process. The usefulness of the method is demonstrated by performing measurements on a standard hydrogenated amorphous silicon sample. (C) 2004 American Institute of Physics. [DOI: 10.1063/1.1818732]
\end{abstract}

In 1986, Ritter et al. ${ }^{1}$ described a technique for measuring the ambipolar diffusion length in low-mobility semiconductors. This technique, called steady-state photocarrier grating (SSPG), became a standard method for material characterization in several laboratories. These authors ${ }^{1,2}$ presented the basic equations describing the phenomenon, and solved them to obtain a simple fitting formula from which the diffusion length could be extracted. The solution, however, included some phenomenological parameters, like the small signal recombination lifetime, and it was based on the assumption of ambipolar transport. Several authors addressed the subject of analyzing theoretical aspects of SSPG, ${ }^{3-6}$ but a controversy about whether ambipolar transport occurred in amorphous semiconductors remained. In 1995, Abel et al. ${ }^{7}$ presented a generalized treatment of SSPG, including bipolar transport and the effects of the external electric field. These authors obtained an analytical expression which could be used as a fitting function for experiments where the grating period $\Lambda$ and the electric field $\xi$ were varied. Despite several works in the field, no attempt to correlate the SSPG results with the density of localized states in the mobility gap has been presented up to the moment. In this letter, we take as starting parameters the density of states (DOS) distribution within the mobility gap, the extendedstates mobility of the carriers, and the capture coefficients. Though we have solved analytically the equations describing the SSPG experiment for DOS distributions including different types of defects (monovalent and/or amphoteric), for the sake of clarity we will concentrate here on the case of a single type of monovalent states. Performing suitable approximations on the analytical solution of the SSPG equations, we obtain a simple formula relating the DOS at the electron quasi-Fermi energy to material parameters and measurable quantities. We verify the simplifying assumptions and the final theoretical expression by comparison with numerical simulations. We prove the applicability of this

${ }^{a)}$ Electronic mail: jschmidt@intec.unl.edu.ar method by performing measurements on a standard hydrogenated amorphous silicon $(a-\mathrm{Si}: \mathrm{H})$ sample.

When two laser beams of different intensities interfere on the sample surface (coordinate $x$ ), an intensity grating $I(x)=I_{1}+I_{2}+2 \sqrt{I_{1} I_{2}} \cos (2 \pi x / \Lambda)$ is created, where the grating period is $\Lambda=\lambda /[2 \sin (\delta / 2)]$, $\lambda$ being the laser wavelength and $\delta$ the angle between the two beams. This leads to a spatially modulated generation rate, $G(x)=G_{0}+\Delta G \cos (k x)$, $k=2 \pi / \Lambda$, that in turn creates free electrons and holes distributions, $n(x)$ and $p(x)$, with the same period. However, since electrons and holes have different diffusion coefficients, the amplitudes and phases of the two distributions will differ, generating an internal electric field, $\xi_{\text {int }}(x)$, that will add to the externally applied electric field, $\xi_{\text {ext }}$. The internal electric field is related to the local charge densities via Poisson's equation

$$
\begin{aligned}
\frac{d \xi_{\text {int }}(x)}{d x}= & \frac{q}{\varepsilon \varepsilon_{0}}\left\{p(x)+\int_{E_{v}}^{E_{c}}[1-f(E, x)] N^{\mathrm{DON}}(E) d E\right. \\
& \left.-n(x)-\int_{E_{v}}^{E_{c}} f(E, x) N^{\mathrm{ACC}}(E) d E\right\},
\end{aligned}
$$

where $\varepsilon$ is the dielectric constant of the sample, $\varepsilon_{0}$ is the dielectric permittivity of vacuum, $E_{v}$ is the energy at the top of the valence band, $E_{c}$ is the energy at the bottom of the conduction band, $f(E, x)$ is the occupation function, $N^{\mathrm{DON}}(E)$ is the density of donor states (neutral when occupied and positively charged when unoccupied), and $N^{\mathrm{ACC}}(E)$ is the density of acceptor states (neutral when empty and negatively charged when occupied). Both the externally applied electric field and the internally developed space-charge field will contribute to the current density under coherent illumination, which is given by 


$$
\begin{aligned}
j_{\mathrm{coh}} & =\frac{1}{\Lambda} \int_{0}^{\Lambda}\left[q \mu_{n} n(x)+q \mu_{p} p(x)\right]\left[\xi_{\mathrm{ext}}+\xi_{\mathrm{int}}(x)\right] d x \\
& =j_{0}+\Delta j .
\end{aligned}
$$

The carrier concentrations $n(x)$ and $p(x)$ are obtained by solving the continuity equations for electrons and holes, which in the steady state are

$$
\begin{aligned}
& G(x)-R_{n}(x)=-\mu_{n} \frac{d}{d x}[n(x) \xi(x)]-D_{n} \frac{d^{2} n(x)}{d x^{2}}, \\
& G(x)-R_{p}(x)=\mu_{p} \frac{d}{d x}[p(x) \xi(x)]-D_{p} \frac{d^{2} p(x)}{d x^{2}},
\end{aligned}
$$

where $\mu$ is the extended-states mobility, and $D$ is the diffusion coefficient. Subscripts ( $n$ or $p$ ) refer to electrons and holes, respectively. The recombination rates $R(x)$ are given by

$$
\begin{aligned}
& R_{n}(x)=\int_{E_{v}}^{E_{c}}\left\{c_{n} n(x)[1-f(E, x)]-e_{n}(E) f(E, x)\right\} N(E) d E, \\
& R_{p}(x)=\int_{E_{v}}^{E_{c}}\left\{c_{p} p(x) f(E, x)-e_{p}(E)[1-f(E, x)]\right\} N(E) d E,
\end{aligned}
$$

where $c$ is the capture coefficient, $e(E)$ is the emission coefficient, and $N(E)$ is the DOS.

In the low-modulation condition established when $I_{1}$ $\gg I_{2}$, it is expected that the relevant physical parameters vary sinusoidally as $G(x)$ does. In general, however, there will be variable phase shifts, and any quantity can be expressed as $Q(x)=Q_{0}+\Delta Q \cos (k x+\varphi)$, where $Q_{0}$ is the value under uniform illumination $G_{0}=G_{1}+G_{2}$, and $\Delta Q$ originates from the spatially modulated term of the generation rate $\Delta G(x)$. This linearizes the system of coupled differential Eqs. (1) and (3)-(6), giving rise to the following system of linear equations

$$
\left\{\begin{array}{c}
A_{n} \times \Delta n \cos \left(\varphi_{n}\right)-B_{n} \times \Delta n \sin \left(\varphi_{n}\right)+A_{p}^{*} \times \Delta p \cos \left(\varphi_{p}\right)=\Delta G \\
B_{n} \times \Delta n \cos \left(\varphi_{n}\right)+A_{n} \times \Delta n \sin \left(\varphi_{n}\right)+A_{p}^{*} \times \Delta p \sin \left(\varphi_{p}\right)=0 \\
A_{n}^{*} \times \Delta n \cos \left(\varphi_{n}\right)+A_{p} \times \Delta p \cos \left(\varphi_{p}\right)-B_{p} \times \Delta p \sin \left(\varphi_{p}\right)=\Delta G \\
A_{n}^{*} \times \Delta n \sin \left(\varphi_{n}\right)+B_{p} \times \Delta p \cos \left(\varphi_{p}\right)+A_{p} \times \Delta p \sin \left(\varphi_{p}\right)=0
\end{array}\right.
$$

The coefficients are given by

$$
\begin{aligned}
A_{n}= & c_{n} \int\left[1-\left(c_{n} n_{0}+e_{n}\right) \tau\right]\left(1-f_{0}\right) N d E \\
& +\frac{q \mu_{n} n_{0}}{\varepsilon \varepsilon_{0}}\left[1+c_{n} \int\left(1-f_{0}\right) \tau N d E\right]+k^{2} D_{n}, \\
A_{n}^{*}= & c_{n} \int\left(c_{p} p_{0}+e_{p}\right) \tau\left(1-f_{0}\right) N d E \\
& -\frac{q \mu_{p} p_{0}}{\varepsilon \varepsilon_{0}}\left[1+c_{n} \int\left(1-f_{0}\right) \tau N d E\right], \\
A_{p}= & c_{p} \int\left[1-\left(c_{p} p_{0}+e_{p}\right) \tau\right] f_{0} N d E \\
& +\frac{q \mu_{p} p_{0}}{\varepsilon \varepsilon_{0}}\left[1+c_{p} \int f_{0} \tau N d E\right]+k^{2} D_{p}, \\
A_{p}^{*}= & c_{p} \int\left(c_{n} n_{0}+e_{n}\right) \tau f_{0} N d E-\frac{q \mu_{n} n_{0}}{\varepsilon \varepsilon_{0}}\left[1+c_{p} \int f_{0} \tau N d E\right], \\
B_{n}= & -k \mu_{n} \xi_{\mathrm{ext}}, \quad \text { and } \quad B_{p}=k \mu_{p} \xi_{\mathrm{ext}},
\end{aligned}
$$

where the energy dependence has been skipped for the sake of clarity. Integrals are evaluated between $E_{v}$ and $E_{c}$, and we call $\tau^{-1}=c_{n} n_{0}+c_{p} p_{0}+e_{n}+e_{p}$.

From the solution of Eq. (7), analytical expressions for $n(x), p(x)$, and $\xi(x)$ can be obtained. Inserting them into Eq.

$$
\begin{aligned}
\Delta j(\Lambda)= & -\frac{q^{2} \Delta n \Delta p \sin \left(\varphi_{n}-\varphi_{p}\right)}{2 k \varepsilon \varepsilon_{0}}\left\{\mu_{n}\left[1+c_{p} \int f_{0} \tau N d E\right]\right. \\
& \left.+\mu_{p}\left[1+c_{n} \int\left(1-f_{0}\right) \tau N d E\right]\right\} .
\end{aligned}
$$

The full expression of $\Delta n \Delta p \sin \left(\varphi_{n}-\varphi_{p}\right)$ is rather complex, but in the high- $\Lambda$ limit, when $\Delta j$ tends toward a constant value, the terms containing $k$ in this expression can be neglected. After some lengthy calculations, and assuming that $\mu_{n} \gg \mu_{p}$, the following limit expression is obtained ${ }^{8}$

$$
\Delta j(\Lambda \rightarrow \infty)=-\frac{1}{2} j_{0}\left(\frac{\Delta G}{G_{0}}\right)^{2} \frac{1}{\left(1+X_{n}\right)^{2}},
$$

where we call $X_{n}=c_{n} \tau_{n} k_{B} T N\left(E_{t n}\right)$, with $\tau_{n}$ the electron recombination lifetime, $k_{B}$ the Boltzmann's constant, $T$ the absolute temperature, and $E_{t n}$ the quasi-Fermi level for trapped electrons. The coefficient $\beta$, defined by Ritter et al. in Ref. 1, can be evaluated in the high- $\Lambda$ limit as

$$
\beta_{\lim }=\frac{j_{\mathrm{coh}}-j_{1}}{j_{0}-j_{1}}=1-\frac{1}{2} \frac{j_{0}}{j_{0}-j_{1}} \frac{\left(\Delta G / G_{0}\right)^{2}}{\left(1+X_{n}\right)^{2}}
$$

where $j_{1}$ is the current density generated by $I_{1}$ (i.e., $G_{1}$ ) alone. Taking into account the power-law dependence of the conductivity on the generation rate, $\sigma \propto G^{\gamma}$, the following expression can be obtained 


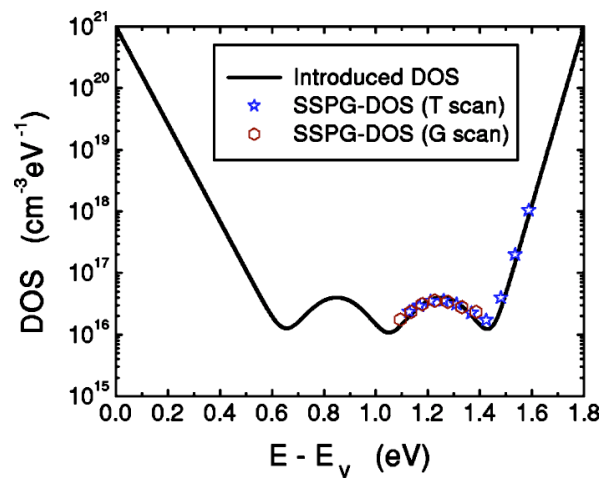

FIG. 1. (Color online) Comparison of the introduced DOS and the calculated one, the SSPG curves being simulated either at different temperatures or at different generation rates.

$$
N\left(E_{t n}\right)=\frac{q \mu_{n} G_{0}}{c_{n} k_{B} T \sigma_{0}}\left[\frac{1}{\left(1+G_{2} / G_{1}\right)} \sqrt{\frac{2 \sigma_{0}}{\gamma \sigma_{1}\left(1-\beta_{\mathrm{lim}}\right)}}-1\right] .
$$

This equation expresses the DOS at the quasi-Fermi energy as a function of material parameters $\left(c_{n}\right.$ and $\left.\mu_{n}\right)$ and experimental magnitudes that can be easily measured (temperature, generation rate, photoconductivity, $\gamma$ and $\beta_{\text {lim }}$ ). The quasiFermi energy $E_{t n}$ can be evaluated from the steady-state photoconductivity $\sigma_{0}$, and it can be varied either from a temperature scan or a generation rate scan. That gives the basis for a DOS spectroscopy in the upper half of the band gap. The experiment can be performed at a single grating period $\Lambda$, provided it is in the high- $\Lambda$ region where the $\beta(\Lambda)$ curve tends toward a constant value. Thus, the experimental setup is simple.

We have performed a numerical simulation of the experiment, starting from a typical DOS for $a-\mathrm{Si}: \mathrm{H}$. We have taken standard values for the material parameters, and we have solved numerically the set of Eqs. (1) and (3)-(6) to get the $\beta(\Lambda)$ curves (which closely resemble the experimental ones). Then we applied Eq. (11) to reconstruct the DOS, changing $E_{t n}$ from a variation of $T$ and $G_{0}$. Figure 1 shows the reconstruction of the initially introduced DOS. As can be seen, the DOS can be reconstructed with quite good accuracy. Similar results have been obtained from several simulations for different DOSs.

To prove the experimental usefulness of the technique, preliminary measurements on an $a-\mathrm{Si}: \mathrm{H}$ sample have been made. We have deposited parallel aluminum contacts $1 \mathrm{~mm}$ apart. We have taken $\Lambda=10 \mu \mathrm{m}$ and we have measured the coefficients $\beta$ and $\gamma$ as a function of temperature and generation rate. We performed a temperature scan from 100 to $370 \mathrm{~K}$ in $30 \mathrm{~K}$ steps; and then at a fixed temperature of 370 $\mathrm{K}$ we varied the generation rate between 2 $\times 10^{19} \mathrm{~cm}^{-3} \mathrm{~s}^{-1}<G_{0}<2 \times 10^{21} \mathrm{~cm}^{-3} \mathrm{~s}^{-1}$. The result is shown in Fig. 2, where our technique based on SSPG mea-

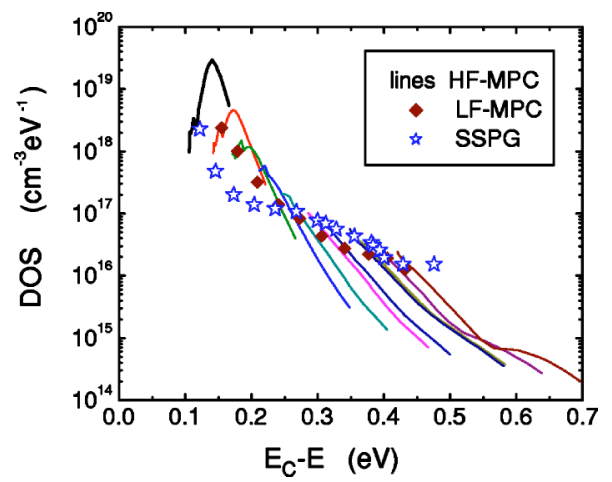

FIG. 2. (Color online) Comparison between DOS determinations on the same $a$-Si:H sample from MPC in the low-frequency (LF-MPC) and highfrequency (HF-MPC) domains, and from SSPG. $N\left(E_{c}\right)=10^{21} \mathrm{~cm}^{-3} \mathrm{eV}^{-1}$, $\mu_{n}=10 \mathrm{~cm}^{2} \mathrm{~V}^{-1} \mathrm{~s}^{-1}$, and $c_{n}=10^{-8} \mathrm{~cm}^{3} \mathrm{~s}^{-1}$ have been assumed in all cases.

surements is compared with the modulated photocurrent (MPC) methods, performed on the same sample both in the high-frequency ${ }^{9}$ and the low-frequency ${ }^{10}$ limits. As can be seen, the agreement is very good, except for energies close to the conduction-band tail. The disagreement in this region may be due to a failure of some of the simplifying hypotheses when the DOS is steeper than $k_{B} T .^{8}$ However, the general agreement between the three DOS estimations means that the SSPG technique provides a simple method for DOS determinations, with an accuracy at least comparable to the one of the MPC techniques.

In conclusion, we have presented a simple method for DOS determinations based on SSPG measurements performed in the high- $\Lambda$ limit. We have performed simulations starting from a DOS typical for $a-\mathrm{Si}: \mathrm{H}$, and we have shown that the method is able to reproduce the introduced DOS. We have presented preliminary measurements on an $a-\mathrm{Si}: \mathrm{H}$ sample, which demonstrate that the method is applicable, experimentally simple, and capable of providing DOS determinations compatible with the ones obtained from the modulated photoconductivity methods.

This work was partly supported by ECOS Sud-SECyT (Project No. A02E01). One of the authors (J.A.S.) also acknowledges support from CONICET (PEI No. 6329) and the Alexander von Humboldt Foundation.

${ }^{1}$ D. Ritter, E. Zeldov, and K. Weiser, Appl. Phys. Lett. 49, 791 (1986).

${ }^{2}$ D. Ritter, K. Weiser, and E. Zeldov, J. Appl. Phys. 62, 4563 (1987).

${ }^{3}$ I. Balberg, J. Appl. Phys. 67, 6329 (1990).

${ }^{4}$ Y.-M. Li, Phys. Rev. B 42, 9025 (1990).

${ }^{5}$ K. Hattori, H. Okamoto, and Y. Hamakawa, Phys. Rev. B 45, 1126 (1992).

${ }^{6}$ J. P. Nicholson, J. Appl. Phys. 88, 4693 (2000).

${ }^{7}$ C.-D. Abel, G. H. Bauer, and W. H. Bloss, Philos. Mag. B 72, 551 (1995).

${ }^{8}$ Details will be given in a forthcoming publication.

${ }^{9}$ C. Longeaud and J. P. Kleider, Phys. Rev. B 45, 11672 (1992).

${ }^{10}$ R. R. Koropecki, J. A. Schmidt, and R. Arce, J. Appl. Phys. 91, 9865 (2002). 